\title{
Neural oscillatory mechanisms during novel grammar learning underlying language analytical abilities
}

\author{
Olga Kepinska ${ }^{1,2, *}$, Ernesto Pereda ${ }^{3,4}$, Johanneke Caspers ${ }^{1,2}$, Niels O. Schiller ${ }^{1,2}$ \\ ${ }^{1}$ Leiden University Centre for Linguistics, Postbus 9515, 2300 RA Leiden, the Netherlands \\ ${ }^{2}$ Leiden Institute for Brain and Cognition, c/o LUMC, Postzone C2-S, P.O. Box 9600, 2300 RC Leiden, the Netherlands \\ ${ }^{3}$ Electrical Engineering and Bioengineering group, Dept. of Industrial Engineering \& Instituto Universitario de \\ Neurociencias, Universidad de La Laguna, Tenerife, Spain \\ ${ }^{4}$ Laboratory of Cognitive and Computational Neuroscience, Centre of Biomedical Technology, Universidad Politécnica \\ de Madrid, Madrid, SPAIN \\ *Corresponding author. Tel. +31 (0)71 527 8530, e-mail: o.kepinska@hum.leidenuniv.nl
}

\begin{abstract}
The goal of the present study was to investigate the initial phases of novel grammar learning on a neural level, concentrating on mechanisms responsible for individual variability between learners. Two groups of participants, one with high and one with average language analytical abilities, performed an Artificial Grammar Learning (AGL) task consisting of learning and test phases. During the task, EEG signals from 32 cap-mounted electrodes were recorded and epochs corresponding to the learning phases were analysed. We investigated spectral power modulations over time, and functional connectivity patterns by means of a bivariate, frequency-specific index of phase synchronization termed Phase Locking Value (PLV). Behavioural data showed learning effects in both groups, with a steeper learning curve and higher ultimate attainment for the highly skilled learners. Moreover, we established that cortical connectivity patterns and profiles of spectral power modulations over time differentiated L2 learners with various levels of language analytical abilities. Over the course of the task, the learning process seemed to be driven by whole-brain functional connectivity between neuronal assemblies achieved by means of communication in the beta band frequency. On a shorter time-scale, increasing proficiency on the AGL task appeared to be supported by stronger local synchronisation within the right hemisphere regions. Finally, we observed that the highly skilled learners might have exerted less mental effort, or reduced attention for the task at hand once the learning was achieved, as evidenced by the higher alpha band power.
\end{abstract}

KEYWORDS. Language learning; EEG; Oscillations; Second language acquisition; Artificial Grammar Learning; Language aptitude

\section{Introduction}

Second language (L2) learning can be characterised by a great deal of variability in the rate, efficiency and ultimate success. For some individuals, it requires strenuous efforts, whereas, for others, high levels of proficiency in an L2 can be attained with relative ease and little time investment. Understanding what factors are responsible for such variability among learners is important both for second language acquisition (SLA) theory building, and the applied efforts for learning and instruction improvements.

From a neurophysiological point of view, high-level cognitive functions such as language, necessarily depend on synchronised activity both between and within specific neural assemblies (Bressler, 1995; Bressler \& Menon, 2010; Friederici \& Singer, 2015; Maguire \& Abel, 2013; Mesulam, 1998; W. Singer, 1993; Varela, Lachaux, Rodriguez, \& Martinerie, 2001; Weiss \& Mueller, 2003). Investigations into interactions taking place in different brain areas and the networks arising from them are invaluable for understanding the neural underpinnings of human communication. One possible way of exploring such interactions is to study the rhythms of the brain, their topographical properties, task- and state-dependent development, and dynamics. Such neural oscillations lie at the centre of 
coordinated activity of the brain and are seen as one of the fundamental mechanisms enabling learning and neural plasticity (Benchenane et al., 2010; Uhlhaas \& Singer, 2010).

The goal of this paper is to investigate the initial phases of learning a new language, when completely new linguistic input is identified, analysed, processed, and - with various levels of success and different degrees of efficiency - learned. We are interested in the way such a learning process is reflected in neural oscillations over time and in the mechanisms responsible for variability between the learners. A technique well suited for investigating brain oscillatory architecture during language learning is electroencephalography (EEG) (cf. De Diego-Balaguer, Fuentemilla, \& Rodriguez-Fornells, 2011; Reiterer, Pereda, \& Bhattacharya, 2009; Wang, 2010). It offers a view on the nature of brain rhythms in different frequencies during information processing, and coherence or synchronisation analyses can be used to visualise the functional cooperation between cortical regions.

EEG signals recorded during a cognitive task can be indicative of synchronisation of local and distant cortical networks; the signal can be quantified by means of, for example, power spectrum or synchronisation analyses (cf. e.g. Siegel, Donner, \& Engel, 2012; Wang, 2010). Spectral power variations reflect the number of neurons discharging at the same time (Kiiski et al., 2012; Klimesch, 1999), and are thus seen as a measure of local neuronal activity. Employing coherence analyses, EEG offers a view on functional cooperation between cortical regions: brain areas activated by a particular cognitive task exhibit increased coherence, and high coherence between two EEG signals is indicative of high cooperation (degree of information flow) and synchronisation between underlying brain regions within a certain frequency band (Weiss \& Mueller, 2003).

Furthermore, there are various putative roles assigned to different frequency bands (see e.g. Uhlhaas \& Singer, 2010 for an overview). For example, the beta band frequency $(13-29 \mathrm{~Hz}$ ) has been linked to long-range synchronisation (Kopell, Ermentrout, Whittington, \& Traub, 2000), and within the language domain, to underlie such higher-order functions as semantic memory and syntactic binding (Bastiaansen, Magyari, \& Hagoort, 2010; Weiss \& Mueller, 2012). Gamma band frequency $(30-40 \mathrm{~Hz})$ is believed to be tied to for example perceptual processing, attention, and episodic memory formation (cf. Herrmann, Munk, \& Engel, 2004; Reiterer et al., 2009). In the context of learning, Miltner, Braun, Arnold, Witte and Taub (1999) found the development of gamma band coherence to be a signature of associative learning. They put forward that an increase in coherence in the gamma band "could fulfil the criteria required for the formation of Hebbian cell assemblies, binding together parts of the brain that must communicate with one another in order for associative learning to take place" (Miltner et al., 1999) and suggested it could be the case for other types of learning as well.

The different EEG frequency bands can also reflect various strategies employed for learning. De Diego-Balaguer et al. (2011) investigated the dynamics of synchronisation properties of the EEG signal during auditory language learning. In a study employing exposure to an artificial language, they found that increased long range gamma band phase coherence between frontal, temporal, and parietal regions accompanied successful learning of rules embedded in the presented speech stream. On the other hand, poor rule learners exhibited greater local synchrony in the gamma range and increased theta-band $(4-8 \mathrm{~Hz})$ coherence over the course of the task. Different learning strategies for the two groups were thus 
suggested based on both behavioural and neurophysiological data. Whereas successful learning was driven by rule extraction (and coupled with long range gamma band coherence), the poor learners seemed to apply a more memoryoriented, template-matching strategy during learning, reflected in local gamma power and theta-band coherence increases.

Within the framework of SLA, coherence of the EEG signals was investigated by Reiterer, Pereda and Bhattacharya $(2009 ; 2011)$ who explored differences in EEG synchronisation measures in the gamma band range between second language speakers of English with high and low proficiency who were listening to first (L1, German) and second language input. The authors reported different patterns of synchronisation for the two groups, involving more extensive networks in the right hemisphere for participants with low L2 proficiency during processing of English input, and an inverse relationship between L2 proficiency and synchronisation density: high proficiency was coupled with lower synchronisation (Reiterer et al., 2009). Further investigation into the gamma band phase synchrony (Reiterer et al., 2011) by means of coarse-graining of Markov chains (Allefeld \& Bialonski, 2007) and phase lag index (Stam, Nolte, \& Daffertshofer, 2007) revealed differences between low and high L2 proficiency participants during second language processing. The group with less L2 expertise displayed stronger and broader network patterns than the high proficiency group, especially in frontoparietal areas of the left hemisphere. The authors noted that differences between the two groups of learners might have reflected pre-existing individual differences in the linguistic abilities of the participants, rather than differences in L2 proficiency only.

Within the field of SLA, such individual differences in linguistic abilities are referred to as language aptitude. Language aptitude is a relatively fixed ability of an L2 learner to acquire a language. It is a strong predictor of achievement in L2 (Dörnyei \& Skehan, 2003; Ellis, 2008; Sawyer \& Ranta, 2001) and plays an important role both in instructed (de Graaff, 1997) and naturalistic (Abrahamsson \& Hyltenstam, 2008; DeKeyser, 2000; Harley \& Hart, 2002; Robinson, 1997) language learning. Traditionally, language aptitude has been operationalised by means of standardised test instruments aiming at capturing learners' abilities underlying L2 acquisition. Such tests typically consist of a number of different parts, underscoring the multi-componential nature of language aptitude of which four sub-components are traditionally distinguished: rote learning memory, phonemic coding ability, inductive language learning ability and grammatical sensitivity, the two latter also referred to as language analytic ability (LAA) (cf. Abrahamsson \& Hyltenstam, 2008; Carroll, 1981; Dörnyei \& Skehan, 2003; Ellis, 2008; Sawyer \& Ranta, 2001; Skehan, 2002). In recent years, the theoretical construct of language aptitude has also been investigated from a neuroscientific perspective, with the aim of indicating the aspects of brain functioning that underlie the different aptitude components (Hu et al., 2013; Kepinska, de Rover, Caspers, \& Schiller, 2017; Prat, Yamasaki, Kluender, \& Stocco, 2016; Xiang, Dediu, Roberts, Norris, \& Hagoort, 2012).

In this study, drawing on the construct of language aptitude, we wanted to a priori control for individual variability between the learners, and explore the neural mechanisms of language learning coupled with high and moderate abilities. In our approach, we focused on one of the most important elements of L2 acquisition, namely novel grammar learning. Our aim was to explore how the sub-component of language aptitude crucial for grammar acquisition (i.e. the LAA), influences initial phases of L2 learning and the properties of the EEG signal measured during 
such a task. To this end, we employed an experimental design previously used in research investigating the neurobiological basis of language acquisition, namely an Artificial Grammar Learning (AGL) paradigm. The design of our experiment was based on the study of Opitz et al. (2011), where the artificial language BROCANTO was used (cf. Friederici et al., 2002; Opitz \& Friederici, 2003). In studies employing the BROCANTO language (e.g. Brod \& Opitz, 2012; Friederici et al., 2002; Hauser, Hofmann, \& Opitz, 2012; Opitz, Ferdinand, \& Mecklinger, 2011; Opitz \& Friederici, 2003, 2004, 2007) participants are presented with sentences constructed according to its rules and are instructed to extract the underlying grammar. The paradigm consists of learning and test phases. During learning, participants are presented only with grammatically correct sentences. In the test phases, both grammatical and ungrammatical sentences are presented and participants are asked to give a grammaticality judgement on the sentences.

Previous fMRI studies employing this paradigm concentrated on temporal changes in brain activity, and on the underpinnings of two types of knowledge acquired over the course of AGL: rule and similarity knowledge. Opitz and Friederici (2003) reported decreasing activity of the hippocampus and increasing activity of the left inferior frontal gyrus as a function of time and performance during the task. Opitz and Friederici (2004) extended these results by suggesting that the hippocampus and right IFG support grammar learning when the acquired knowledge is based on similarity; the left ventral premotor cortex was coupled with rule knowledge (Hauser et al., 2012; Opitz \& Friederici, 2004). Furthermore, a recent experiment from our group investigating individual differences in grammar learning (Kepinska et al., 2017) pointed to right fronto-parietal involvement underlying superior skills for grammar learning. EEG studies using the BROCANTO task have concentrated on the event-related potentials (ERP) coupled with adult 'native-like' processing of the newly learned grammar (Friederici et al., 2002), and signatures of feedback processing during the learning task (Opitz et al., 2011).

In the current study, we investigated whether learners with different degrees of LAA exhibit different oscillatory patterns during acquisition of a novel grammar, as operationalized by the BROCANTO AGL paradigm. Furthermore, we are interested in the dynamics of the learning process of a novel grammar and in the way the learning process is represented on a neural level, namely in short- and long-range brain oscillations. Our analysis is based on the approach of Reiterer et al. (2011) and De Diego-Balaguer et al. (2011). Based on their work, our hypothesis is that synchronisation mechanisms play a key role during language learning and that those mechanisms are modulated by individual differences in pre-tested language learning abilities. On the basis of Miltner et al. (1999), we expect an increase of phase synchrony values as the learning proceeds in the high frequencies (30-40 Hz corresponding to the gamma band). Furthermore, following the results of De Diego-Balaguer et al. (2011), we expect stronger high frequency (30-40 Hz) long-range synchrony among the highly skilled learners; lower analytical abilities are hypothesised to be coupled with local high frequency (30$40 \mathrm{~Hz}$ ) synchrony and power. Due to the importance of the low frequency oscillations (4-8 $\mathrm{Hz}$, corresponding to the theta band) for lower level memory processes during learning, we expect the synchrony in low frequency to be tied with initial phases of grammar learning, with decreasing levels of synchronisation for this band as learning proceeds, especially among the participants with high analytical abilities. 


\section{Materials and methods}

\subsection{Pre-test}

Two groups of participants (with high and average LAA) were determined by administering a language aptitude test to a large group of participants $(N=307)$. We used the Llama Language Aptitude Test (LLAMA) (Meara, 2005), a computerbased test battery with automated scoring, suitable for participants with various language backgrounds. The test consists of four parts: (1) a vocabulary learning task, (2) a test of phonetic memory, (3) a test of sound-symbol correspondence and (4) a test of grammatical inferencing (LLAMA_F), being a measure of LAA. Recruitment of participants for the study was based on the scores on the LLAMA_F test.

In this test, twenty pictures are presented together with sentences in an unknown language that describe them. In the learning phase lasting five minutes, participants learn the grammatical rules, while taking notes. In the test phase, they are presented with a picture and two sentences and have to judge which sentence is grammatically correct. Participants can score from 0 to 100 , where 80 - 100 is defined as outstandingly good and 25 - 45 as average (Meara, 2005).

\subsection{Participants}

Having taken the LLAMA test, a total of 45 healthy, right-handed native speakers of Dutch were invited for the EEG experiment. Three participants were excluded due to technical difficulties. The remaining 42 participants (14 male) were 18-29 years old. On the LLAMA_F test, the participants scored either within average range (i.e. 20 - 50) ${ }^{1}$ (henceforth Average LAA) or got the "outstandingly good" score (i.e. 80 - 100) (henceforth High LAA).

There were 20 participants in the Average LAA group ( 5 male), age $19-25$ years ( $M$ $=22$ years) and 22 participants in the High LAA group (9 male), age 18-29 years ( $M=23$ years). A chi-squared test of the male/female ratio across the two groups was not significant (Chi-squared $=1.1932, \mathrm{df}=1, p=0.27$ ). Groups did not differ significantly in terms of age $\left(t_{(40)}=1.92, p=0.062\right)$.

Testing was conducted according to the Ethical Code of the Faculty of Humanities at Leiden University. Participants gave written informed consent prior to the experiment and were remunerated for their time after the last visit in the lab.

\subsection{Stimuli and design}

The stimulus material was created on the basis of the artificial grammar of BROCANTO (Brod \& Opitz, 2012; Friederici et al., 2002; Hauser et al., 2012; Opitz et al., 2011; Opitz \& Friederici, 2003, 2004, 2007) which follows universal rules of natural languages; a sentence (S) consists of a noun phrase (NP) and a verb phrase (VP) and the different categories are represented by different members: nouns ("gum", "trul", "plox", "tok"), verbs ("pel”, "prez", "glif", "rix"), adjectives ("böke"), adverbs ("rüfi") and determiners ("aaf", always followed by a noun and "aak",

\footnotetext{
1 Although the LLAMA manual defines "average score" as 20-45, a score of 50 was also included as average in this study. The scores are awarded at intervals of 10 and a score of 45 is not possible to obtain.
} 
always followed by a modifier). Twelve possible sentence structures were included, see Table 1.

The task consisted of 15 blocks of learn and test phases, all presented visually. Following the BROCANTO rules, 50 grammatical sentences were formed for the learning phases. A further 150 grammatical sentences were formed for the test phase. The ungrammatical sentences for the test phase contained syntactic violations and were created by substituting words from one category by words from a different category. The violations were either determiner-noun-agreement violations (i.e., $\mathrm{DN}$ instead of $\mathrm{dN}$ and $\mathrm{dMN}$ instead of $\mathrm{DMN}$ ), word class repetitions of nouns or verbs and phrase structure violations (i.e., NP NP and NP NP VP rather than NP VP and NP VP NP, respectively).

\begin{tabular}{l|l} 
Sentence structure & Example sentence \\
\hline $\mathrm{dNv}^{2}$ & aaf gum pel \\
\hline $\mathrm{dNvdN}$ & aaf gum pel aaf gum \\
\hline $\mathrm{dNvDMN}$ & aaf tok rix aak böke gum \\
\hline $\mathrm{dNvmDMN}$ & aaf plox glif rüfi aak böke gum \\
\hline $\mathrm{dNvm}$ & aaf gum prez rüfi \\
\hline $\mathrm{dNvmdN}$ & aaf gum glif rüfi aaf trul \\
\hline $\mathrm{DMNv}$ & aak böke plox pel \\
\hline $\mathrm{DMNvdN}$ & aak böke trul pel aaf trul \\
\hline $\mathrm{DMNvDMN}$ & aak böke gum pel aak böke gum \\
\hline $\mathrm{DMNvmDMN}$ & aak böke plox pel rüfi aak böke plox \\
\hline DMNvm & aak böke plox rix rüfi \\
\hline DMNvmdN & aak böke tok glif rüfi aaf tok
\end{tabular}

Table 1 Twelve possible BROCANTO sentence structures included in the experiment.

During each learning phase, 20 sentences out of a pool of 50 that followed the BROCANTO rules were randomly presented for 7 seconds each. Over fifteen blocks, sentences were presented multiple times, but not within a block. During each test phase, 10 unique correct and 10 unique incorrect sentences were randomly presented. Each presentation lasted until the participant gave a response, or for a maximum of 7 seconds. Participants were asked to indicate by pressing a response box button (left or right) whether the sentence followed the BROCANTO rules or not. Assignment of buttons to grammatical/ungrammatical responses was counterbalanced. $500 \mathrm{~ms}$ after each response, participants received visual feedback lasting for $500 \mathrm{~ms}$, which displayed either the word 'correct' in blue or the word 'incorrect' in red.

\footnotetext{
${ }^{2} \mathrm{~N}=$ noun, $\mathrm{v}=$ verb, $\mathrm{M}=$ adjective, $\mathrm{m}=$ adverb, $\mathrm{d}=$ determiner (followed by a noun) and $\mathrm{D}=$ determiner (followed by a modifier)
} 
In order to observe the learning effects and retention of grammatical rules of BROCANTO, participants were asked to perform a transfer test seven days after the experiment. In the transfer test, they were presented with 200 sentences, half of which following the BROCANTO rules, the other half containing one of the three types of grammatical errors. Each sentence was presented for a maximum of 7 seconds, during which time participants were asked to indicate whether the sentence was grammatically correct or not. In order to prevent further learning, no feedback was provided. Participants' performance on the post-test is reported together with the results of the 15 test phases completed during the EEG recording. Two participants (one from each group) did not perform the post-test.

Both the acquisition task and transfer test were created in E-Prime 2.0.10 software (Psychology Software Tools, 2012) and performed on a desktop computer. Stimuli were presented on a 22 " Philips Widescreen LCD monitor $(1680 \times 1050$ at $60 \mathrm{~Hz})$. Participants were seated at a distance of approximately $60 \mathrm{~cm}$ from the screen. All stimuli were presented in black letters (Courier New, size 20) on an E-Prime 'silver' $(192,192,192)$ background. Due to time constraints for the transfer test, one participant performed a web-based version of the transfer test using Qualtrics (Qualtrics, 2013).

\subsection{EEG recording and pre-processing}

The EEG signals were recorded continuously during the entire AGL task. Recording was performed through $32 \mathrm{Ag} / \mathrm{AgCl}$ cap-mounted electrodes using the Biosemi Active Two recording system (see Figure 1). Eye movement and blink artefacts were recorded as horizontal and vertical electrooculograms (HEOG and VEOG) using bipolar recording from electrodes situated above and below the left eye and outside the outer canthi of both eyes. EEG signals were recorded using ActiView software at $512 \mathrm{~Hz}$. Low-pass filter was set to $100 \mathrm{~Hz}$, high-pass filter to $0.16 \mathrm{~Hz}$ and the CMS was used as reference.

Pre-processing of the data was conducted in EEGLAB version 13.4.4b. Data were filtered in band-pass $0.3-48 \mathrm{~Hz}$ and re-referenced to mastoids. For further analysis, only epochs from the learning phases were selected. Artefact-free epochs were chosen manually after careful visual inspection. One data-set (from the Average LAA group) did not yield any artefact-free epochs and had to be discarded. Independent Component Analysis (ICA) was conducted on the epoched data and components corresponding mainly to vertical eye movements, heart rate variability and noise ( $\leq 3$ components) were removed. The epochs used in the subsequent analysis corresponded to the presentation of BROCANTO sentences and were 7 seconds long (3,584 samples).

\section{EEG data analysis}

Our goal was to investigate the oscillatory mechanisms coupled with language analytical abilities during language learning representing both local and largescale communication between neuronal assemblies. To this end, we investigated two aspects of the EEG signal: spectral power, reflecting local activations of large groups of neurons; and phase synchronisation between different EEG signals, indicating functional relationship between (distant) brain sites (cf. Pérez, Carreiras, Gillon Dowens, \& Duñabeitia, 2015). 


\subsection{Power spectrum analysis}

We estimated the power spectral density of each EEG channel by dividing each epoch in non-overlapping segments of 256 samples and tapered them with a Hamming window. We then calculated the discrete Fourier Transform (DFT) of the sum of these tapered segments and took the amplitude of the sum of the DFTs as the power of the signal in the whole frequency band analysed.

The power spectrum analysis was centred in four commonly studied frequency bands, namely $4-8 \mathrm{~Hz}$ (corresponding to the theta band), 8-12 Hz (corresponding to the alpha band), $13-29 \mathrm{~Hz}$ (corresponding to the beta band), and $30-40 \mathrm{~Hz}$ (corresponding to the gamma band). We decided not to define the alpha bands based on the individual alpha peaks but made sure that they fell within the range for this band as defined above. We then evaluated the changes in power by averaging the data of each frequency according to the bands described and analysed it as a function of the learning process.

\subsection{Functional connectivity}

Phase synchronisation (PS) between all possible electrode pairs $[(32 \times 31) / 2=336$ different electrode pairs] was calculated in the same four frequency bands as for power spectrum analysis. To evaluate the degree of PS between pairs of electrodes, the epochs recorded during learning phases were analysed employing a bivariate, frequency-specific index of PS termed Phase Locking Value (PLV, (Mormann, Lehnertz, David, \& Elger, 2000).

The estimation of PLV is a two-step procedure that consists of first calculating the phases from the EEG electrodes and then using the relative phase between any two electrodes to estimate the degree of PS between them as well as the significance of the index itself (see Supplementary Materials for details).

\subsubsection{Defining electrode clusters from PLV values}

In order to explore the variations in PS in each frequency band as a function of grammar learning, as well as the topographical distribution of these effects, we identified synchronisation clusters consisting of groups of electrodes displaying similar PS profiles. Different methods have been proposed for this aim, all of them based on algebraic manipulation of the matrix of PLV indices (Allefeld \& Bialonski, 2007; Allefeld \& Kurths, 2004). Here, we applied instead an efficient, data-driven clustering method termed affinity propagation (Frey \& Dueck, 2007), which allows the estimation of clusters from the matrix of their bivariate similarities without the need of setting a priori the number of clusters. For this, we estimated the grand average (across participants and frequency bands) of the PLV matrices and used it to estimate the PS clusters, which resulted in six different groups of electrodes showed in Figure 1. Note that the regions so defined, while clearly resembling the usually defined scalp regions of interest based on brain topography (namely, frontal, central, etc.), also shows a certain hemispheric asymmetry, with central, parietal, temporal and occipital electrodes clustered differently in the two hemispheres. 


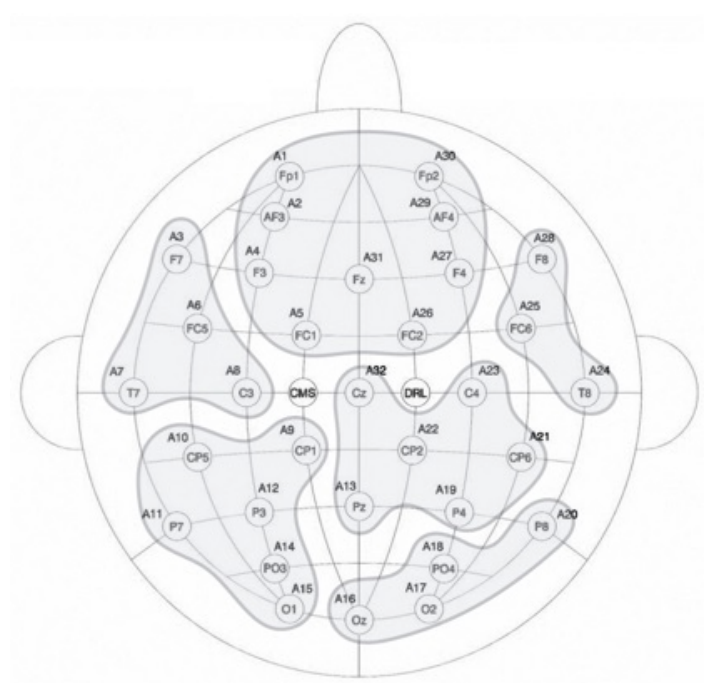

Figure 1 Clusters of electrodes with similar PS profiles used for subsequent analysis of the PLV values overlaid on a schematic representation of the Biosemi Active Two recording system with 32 electrodes.

\subsection{Statistical Analyses}

In order to trace the temporal changes in spectral power over the course of the artificial grammar learning process, we divided the AGL test blocks into four phases of three test blocks each (i.e., \pm 60 sentence trials), and regarded the first block of the task as baseline activity. In order to keep the number of blocks per phase constant, and to avoid effects potentially driven by fatigue instead of the learning process itself, the last two blocks of the task were not subject to the analysis ${ }^{3}$.

Changes in global power values over the course of the task were analysed with a repeated measures ANOVA (alpha level $=0.05$ ) with the between-subject factor group (High and Average LAA) and the within-subject factor learning phase (baseline, first, second, third, fourth phase). When necessary, degrees of freedom were corrected using the Greenhouse-Geisser epsilon value.

The statistical analysis including topographical effects in power and PS values between the two groups of participants concentrated on increases/decreases in power, or PS values across bands, clusters of electrodes and set of blocks (four phases of three blocks each, see above).

For the power and the PS values at the cluster/electrode level we used a paired $t$ test to detect, at the $p<0.05$ level, the existence of significant within-group differences induced by the learning tasks by comparing the original (i.e., base level) block with the averages of blocks 2-4, 5-7 and 8-10 and 11-13 (see Figure 3 and Figure 5). Between-group differences were tested using a t-test for independent samples to compare, for these same blocks, the distribution of power and PS values for the High and Average LAA groups. Finally, we applied, in both cases, the type I false discovery rate (FDR) correction for multiple comparisons (Benjamini \& Yekutieli, 2001) at the $q<0.05$ level t. Of note, the effects we found were

\footnotetext{
${ }^{3}$ In any case, we tested for both the power and the PS indices, the existence of differences between Blocks 11-13 and 14-15, and found that the pattern of between-group differences did not differ between these two sets of blocks.
} 
confirmed, in all cases reported, by applying also the Wilcoxon signed-rank and the U-Mann-Whitney nonparametric tests to analyse within and between group differences, respectively. The use of nonparametric tests along with the FDR correction guarantees that the effects we detect were robust.

Changes in global, whole brain PS values over time were investigated with a repeated measures ANOVA. Furthermore, in order to establish the relationship between the individual performance on the AGL task and the global PS values in the four frequency bands, we performed a regression analysis accounting for the fact that the measurements within the same participant were correlated, i.e. a multilevel analysis of change.

\section{Results}

\subsection{Grammar learning task}

Based on the individual hit- and false-alarm rates, the responses on the AGL task for each participant were transformed into d' scores (Macmillan \& Creelman, 2005). The d' scores were calculated by subtracting the normalised false-alarm rates from normalised hit rates. Missing values in the behavioural data set in four instances (two post-test cases and instances of malfunction of the response buttons, in total 6 cells) were replaced with series means. Scores on the AGL task in both groups increased over the course of the experiment. A repeated-measures ANOVA on the d' scores on the consecutive test blocks and the subsequent posttest showed a main effect of block, $\left(F_{(7.99,319.44)}=20.98, \quad p<.001, \eta_{p}{ }^{2}=0.34\right)$. Furthermore, there was a significant effect of group, $\left(F_{(1,40)}=18.36, p<.001\right.$, $\left.\eta_{\mathrm{p}}{ }^{2}=0.32\right)$ and a significant interaction between group and block $\left(F_{(7.99,319.44)}=3.25, p=.001, \eta_{\mathrm{p}}^{2}=0.075\right)$. The High LAA group performed better than the Average LAA group and showed a higher increase in the proportion of the correct responses than the Average LAA group. Their learning curve was steeper, especially between the $5^{\text {th }}$ and the $8^{\text {th }}$ block of the task (see Figure 2). 


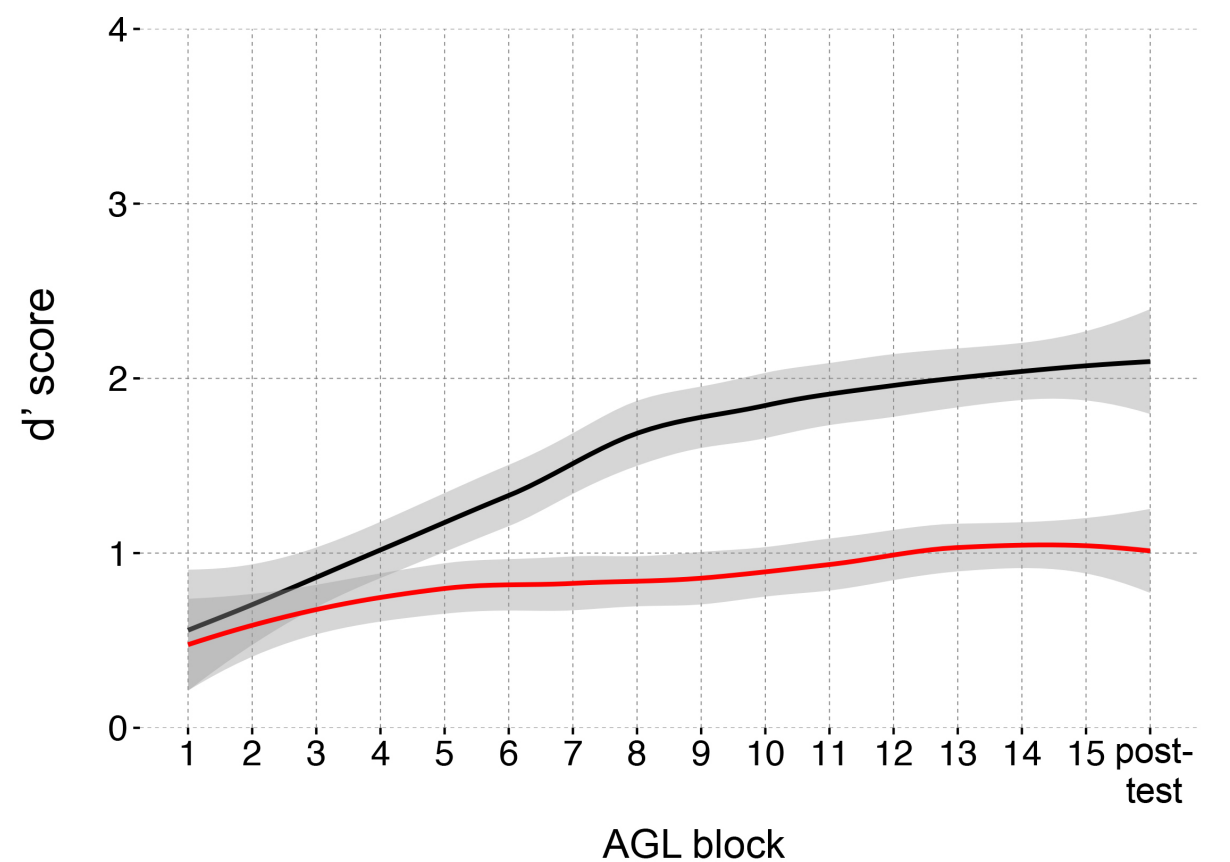

- High LAA — Average LAA

Figure 2 Performance (with 95\% confidence intervals) across participants in High LAA and Average LAA groups during fifteen AGL test phases and the subsequent post-test.

\subsection{Power spectra analysis}

Analysis of power spectra in the theta $(4-8 \mathrm{~Hz})$, alpha $(8-12 \mathrm{~Hz})$, beta $(12-29 \mathrm{~Hz})$, and gamma $(30-40 \mathrm{~Hz})$ bands recorded during the consecutive phases of the learning task was aimed at detecting changes of local oscillatory activity and differences between the High and Average LAA groups.

The analysis revealed modulations with time for theta and alpha band and interactions between factors time and group for beta and gamma bands. A repeated measures ANOVA on global power values showed a main effect of Phase for theta $\left(F_{(1.54,58.74)}=6.70, p=.005, \eta_{p}{ }^{2}=0.15\right)$ and alpha $\left(F_{(2.39,90.82)}=7.15\right.$, $\left.p=.001, \mathrm{n}^{2}=0.16\right)$ bands. Both theta and alpha bands significantly increased linearly $\left(F_{(1,38)}=8.56, p=.006, \mathrm{n}_{p}{ }^{2}=0.18\right.$ and $F_{(1,38)}=13.76, p=.001, \mathrm{n}_{p}{ }^{2}=0.27$, respectively); a less pronounced but significant quadratic increase in alpha band was also observed $\left(F_{(1,38)}=5.25, p=.028, \mathrm{n}^{2}=0.12\right)$.

There was no main effect of Phase for beta and gamma bands (in both cases $p>$.05). In both instances, however, a modulation of the increase by the factor group was observed. There was a significant quadratic increase in beta band power over time $\left(F_{(1,38)}=5.42, p=.025, \mathrm{n}_{p}^{2}=0.13\right)$ and a cubic increase modulated by the factor group $\left(F_{(1,38)}=6.58, p=.014, \mathrm{\eta}^{2}=0.15\right)$. Power in gamma band increased significantly in a cubic mode only in interaction with the factor group $\left(F_{(1,38)}=4.09\right.$, $\left.p=.05, \mathrm{n}_{p}^{2}=0.1\right)$. The power increase in beta and gamma band over time modulated by the factor group can be clearly observed in Figure 3: in case of High LAA learners, the increase reached its maximum in the second phase of the task (blocks 2-4) and decreased afterwards, whereas, after an initial decrease among the Average LAA learners, it kept increasing until the end of the task. 
We further performed a topographical analysis of the 32 electrodes using FDRcorrection for multiple comparisons. The only significant difference between the High and the Average LAA groups (across bands, electrodes and set of blocks at the 0.05 level) was found in alpha band power values and was localised at the occipital electrodes. Corrected $p$-values for each group separately were obtained across bands, electrodes and set of blocks at the 0.05 level only for theta band power. Among the High LAA group, we observed an increase in theta power at the right parieto-occipital and frontal electrodes at the end of the task; significant increases in theta power among the Average LAA group were observed at the occipital electrodes at the beginning of the AGL task, and at the right frontal electrodes towards the end of the task. No significant interactions either with scalp locations (all $p>.05$ ) or power evolution during the learning process (interaction between block and topographical factors, in all cases $p>.05$ ) were observed for other bands. The topographical representation of each band power throughout the AGL task and average power evolvement for all electrodes throughout the task is presented in Figure 3. 


\section{SPECTRAL POWER DURING GRAMMAR LEARNING}

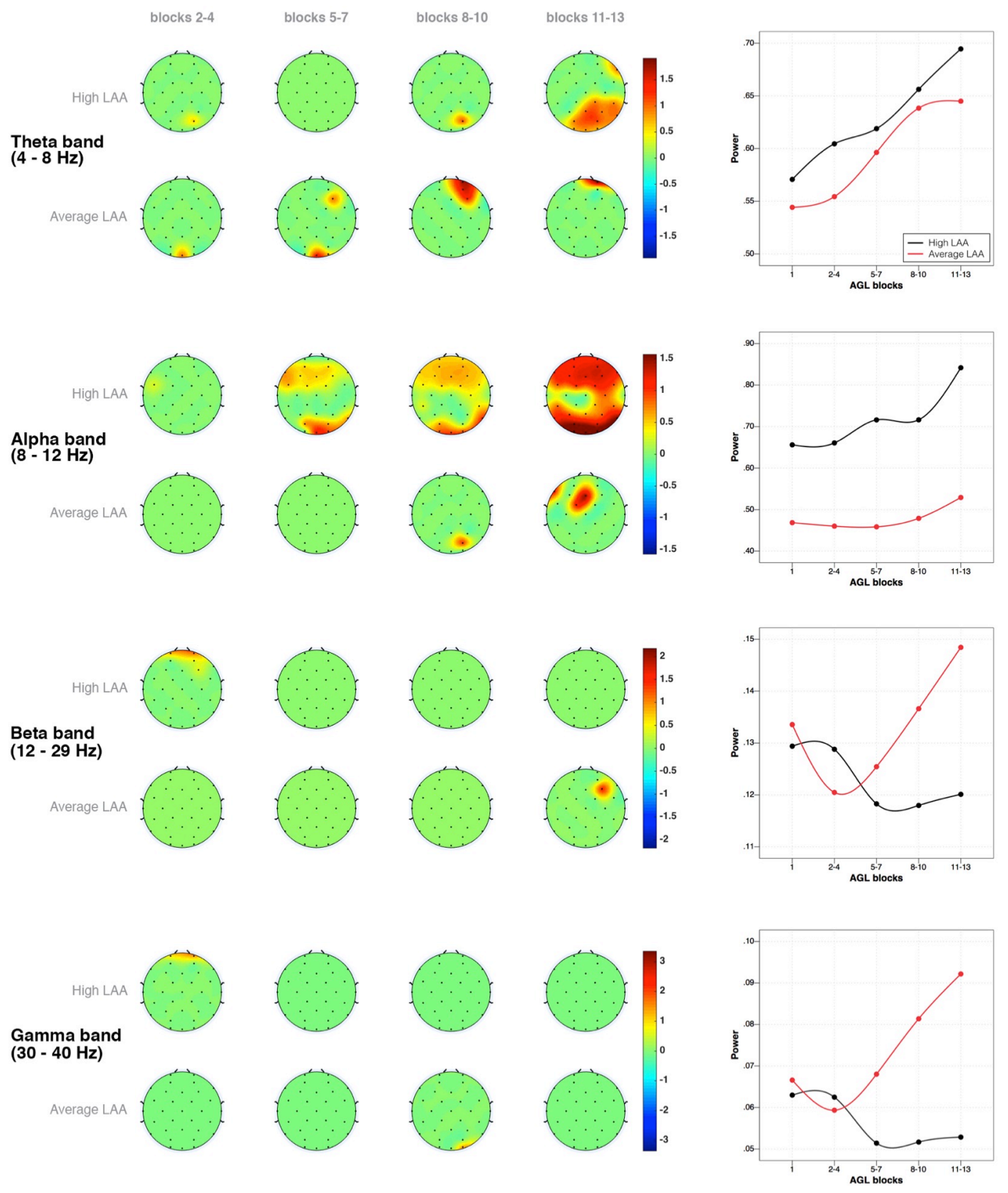

Figure 3. Spectral power modulations as a function of language exposure during the AGL task. The topographical plots on the left present differences between grand mean theta, alpha, beta, and gamma scalp topography spectral power distribution at the selected blocks of the task and the first block. The graphs on the right present mean theta, alpha, beta, and gamma band power evolvement throughout language learning for both groups of participants (in black and red colour for the High and Average LAA groups, respectively) measured at the selected blocks and averaged across all electrodes. 


\subsection{Phase synchrony analysis}

\subsubsection{Global PS values per band and behavioural performance}

The global PS changed over the course of the AGL task. A repeated-measures ANOVA on the PLV values recorded during the consecutive blocks of the learning task showed a main effect of block for theta, alpha, and gamma bands $\left(F_{(8.40,327.44)}=2.91, \quad p=.001, \quad \eta_{p^{2}}=0.07, \quad F_{(7.64,297.86)}=2.74, \quad p=.007, \quad \eta p^{2}=0.07\right.$, $F_{(2.71,105.72)}=3.07, \quad p=.036, \quad \eta_{p}^{2}=0.07$, respectively). Furthermore, a set of exploratory analyses revealed that the amount of global PS in all frequency bands correlated with the performance on the AGL task. On the whole (across all participants), we observed a negative correlation between global PLV values of the slow frequency bands (theta, $r(13)=-.60, p=.001$, and alpha, $r(13)=-.68, p=.005$ ) and d' scores on the learning task, and a positive correlation between the high frequency bands (gamma, $r_{(13)}=.77, p=.001$, and beta, $r(13)=.67, p=.007$ ) and the scores (see Supplementary Figure 1). According to a stepwise linear regression analysis, out of the four frequency bands, only the gamma band global PLV values proved predictive of the performance scores $\left(F_{(1,14)}=19.06, p<.001, R^{2}=.595\right)$.

In order to gain a more fine-grained insight into the relationship between the individual performance on the AGL task and the PS values in the four frequency bands, we performed a regression analysis in which the evolution of AGL d' scores over time was studied as a function of group and the four frequency bands. As the data are longitudinal, measurements within the same subject are correlated. To account for this, a multilevel analysis of change (J. D. Singer \& Willett, 2003) was conducted. For this analysis, our interest centred on the question of which frequency bands contributed most to the behavioural performance during learning, and whether the relationship between the synchronisation values and AGL d' scores differed between the two groups of participants.

The d' scores on the consecutive blocks of the AGL task served as a dependent variable for the analysis; time (block), group (High and Average LAA), and global PLV values in theta, alpha, beta and gamma bands were entered as predictors; we used a random intercept model. To identify a good fitting model, we pursued the following procedure. First, we fitted a model with the main effects of the six predictors, the two-way interactions between them, and the three-way interactions between the predictors (time, group and the four frequency bands). Next, we looked for a parsimonious model by, first, excluding the insignificant three-way interactions, and, next, further removing the insignificant two-way interactions, which were not involved in any significant three-way interaction. The last model resulted in the model with the best fit (as assessed by the Akaike's Information Criterion (AIC), (Akaike, 1973)) and is presented in Table 2. 


\begin{tabular}{|l|r|r|r|r|r|}
\hline \multicolumn{1}{|c|}{ Parameter } & Estimate & \multicolumn{1}{c|}{$\mathrm{SE}$} & \multicolumn{1}{c|}{$\mathrm{df}$} & \multicolumn{1}{c|}{$\mathrm{t}$} & \multicolumn{1}{c|}{ Sig. } \\
\hline Intercept & -.27 & \multicolumn{1}{c|}{1.83} & 250.17 & -.147 & .884 \\
\hline Alpha & 1.15 & 1.93 & 563.85 & .597 & .551 \\
\hline Beta & -.03 & 3.94 & 390.51 & -.007 & .994 \\
\hline Gamma & -.52 & 1.43 & 567.38 & -.360 & .719 \\
\hline Theta & .58 & 1.67 & 425.09 & .345 & .730 \\
\hline Time & -.25 & .14 & 579.68 & -1.789 & .074 \\
\hline Group & -2.59 & 2.43 & 308.14 & -1.066 & .287 \\
\hline group * time &. $\mathbf{4 6}$ & .19 & $\mathbf{5 8 6 . 5 4}$ & $\mathbf{2 . 2 3 8}$ & .026 \\
\hline beta * group & 4.74 & 4.86 & 311.10 & .974 & .331 \\
\hline beta * time &. $\mathbf{7 4}$ & .28 & $\mathbf{5 8 0 . 9 9}$ & $\mathbf{2 . 6 4 6}$ & .008 \\
\hline beta * group * time & -.96 & .37 & $\mathbf{5 8 7 . 6 6}$ & $\mathbf{- 2 . 5 7 4}$ & .010 \\
\hline
\end{tabular}

Table 2 Summary of the results of the multilevel analysis of change: the significant predictors of the AGL task scores are printed in bold font.

Out of the four frequency bands, only the PLV values in beta band proved to be significantly predictive of the AGL task scores over time. In other words, the magnitude of the change of the AGL d' scores over time depended on the global phase synchronisation in beta band. Moreover, the effect of global beta PLV values on the evolution of d' scores during the task was different for the two groups, as evidenced by the significant three-way interaction term between PLV values in beta band, time and group: The time-varying effect of global beta band phase synchronisation on AGL d' scores was larger for the High LAA participants than for the Average LAA participants.

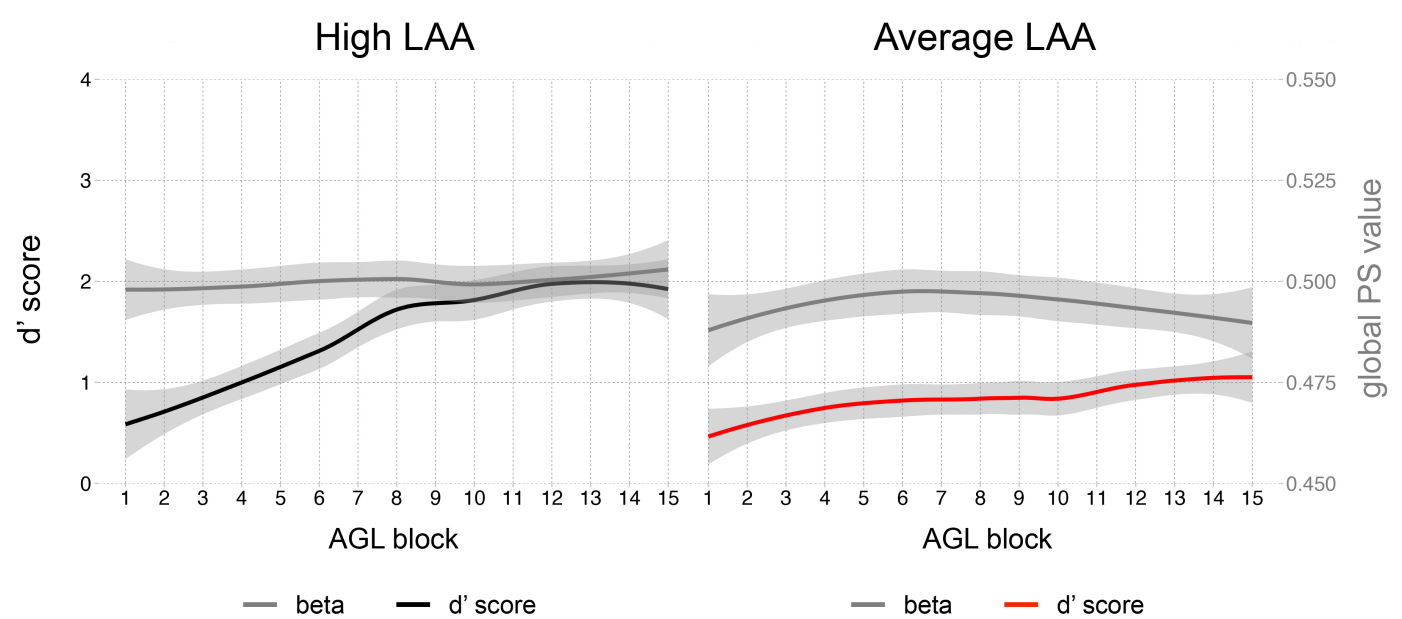

Figure 4 Global PS (PLV) values (surrounded by 95\% Confidence Intervals) in the beta band frequency during the fifteen AGL task blocks plotted against the respective mean AGL task scores, separately for the High and the Average LAA groups. 
This effect can be observed in Figure 4, where the PS values in the beta band recorded during consecutive blocks of the AGL task are plotted against the respective d' scores. An overall higher level of beta band PS values was recorded for the highly skilled learners. Moreover, the relationship of the beta band synchronisation with d' scores varied more over time in case of the High LAA group: the coupling between the PS values and the respective d' scores was stronger in the second half of the task when the behavioural performance was the highest.

\subsubsection{PLV synchrony clusters}

The analysis of the PS within and between the six previously established clusters was performed using FDR-correction for multiple comparisons. For intra-cluster connectivity corrected $p$-values in between-group comparisons were obtained across bands, clusters of electrodes and set of blocks at the 0.05 level for alpha, theta and gamma bands, each time revealing an increase of intra-cluster PS in time larger for the High than for the Average LAA participants (see Figure 5). For theta band, the cluster consisted of right frontal electrodes (F8, FC6, T8), whereas both for alpha and gamma bands, the effect was localised in the right centroparietal regions $(\mathrm{Cz}, \mathrm{Pz}, \mathrm{CP} 2, \mathrm{C} 4, \mathrm{P} 4, \mathrm{CP} 6)$. Notably, in all cases of an increase of intra-cluster PS in time significantly larger for the High than for the Average LAA participants, the effect was observed in the same time-frame, namely in blocks 57.

\section{INTRA-CLUSTER PS DURING GRAMMAR LEARNING High LAA > Average LAA}

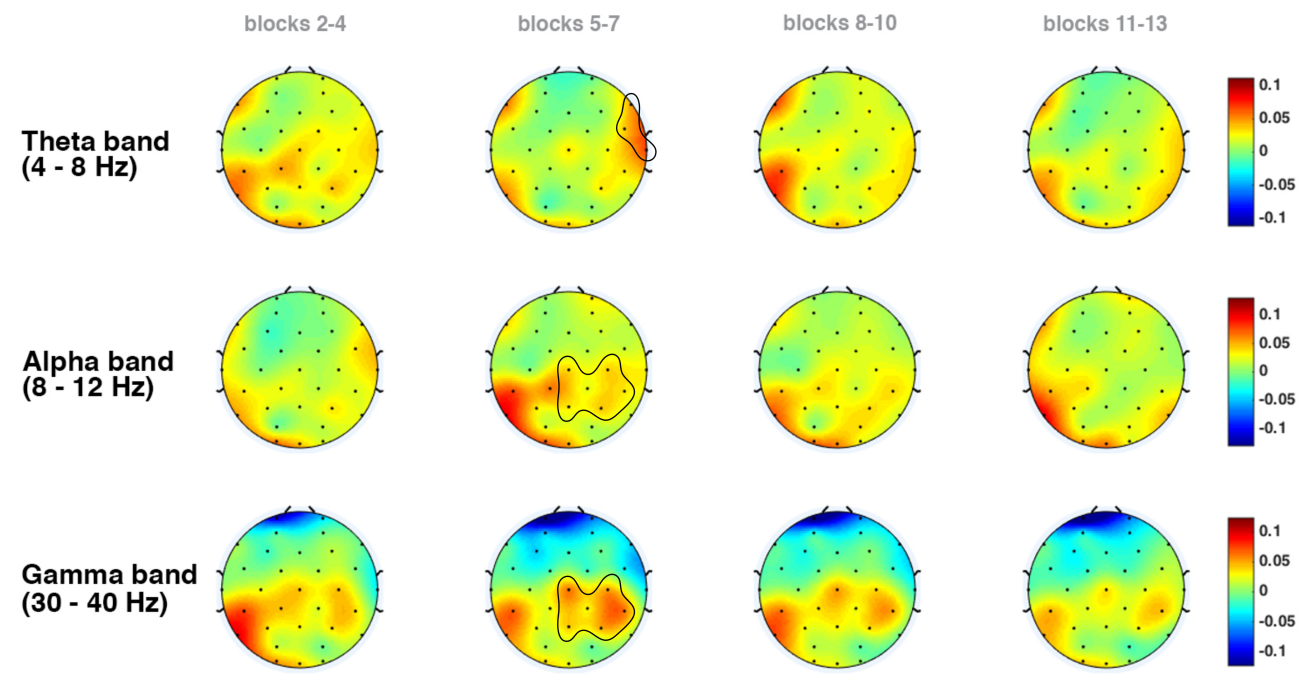

Figure 5. Topographical plots representing differences between mean theta, alpha, beta, and gamma scalp topography PS intra-cluster connectivity distribution at the selected blocks of the task and the first block as a result of a between group comparison (High vs. Average LAA groups). Clusters of electrodes showing increase of intra-cluster PS in time significantly larger for the High than for the Average LAA participants ( $p<.05$, FDR-corrected) are indicated by the black line.

No group differences were observed for the long range, inter-cluster connectivity (all $p>.05$ ). However, the analysis revealed that the PS values were modulated by time in the alpha (both groups) and theta band (only the Average LAA group). In 
alpha band, for the High LAA group, we observed decreases in inter-cluster connectivity from baseline at the beginning of the task (blocks 2-4) at frontal electrodes (Fp1, AF3, F3, FC1, Fp2, AF4, F4, FC2 and Cz) and in the middle of the task (blocks 8-10) in the left frontal region (F7, FC5, C3, T7). The Average LAA group displayed decreases in inter-cluster connectivity from baseline in the middle of the task (blocks 8-10) in the left (CP1, CP5, P3, P7, PO3, O1) and right (P8, PO4, $\mathrm{O} 2, \mathrm{Oz}$ ) parieto-occipital regions. In theta band, for the Average LAA group, we observed a decrease in inter-cluster connectivity from baseline at the beginning of the task (blocks 2-4) in the left parieto-occipital electrodes (CP1, CP5, P3, P7, PO3, O1).

\subsection{Summary of results}

In summary, the obtained results indicated that both groups of participants increased their performance in the grammar learning task, but the group with high language analytical abilities outperformed the moderately skilled learners (see Figure 2). This difference observed on the behavioural level was coupled with several oscillatory mechanisms on the neural level. First, investigating local neural oscillations operationalised by the power spectra analysis, we noted a significant group difference in the alpha power at posterior electrodes towards the end of the task, and different profiles of development over time of the global power spectra in four frequency bands across the two groups (see Figure 3). Furthermore, our analysis of phase synchrony revealed different contributions of the low and high frequency bands over the course of the task to learning. Whereas the theta and alpha bands decreased, as the learning proceeded, beta and gamma increased (see Supplementary Figure 1). What is more, the global PS values in the beta band proved to significantly predict the AGL d' scores over the course of the task as a function of language analytical abilities: the effect of global beta band phase synchronisation on AGL d'scores over time was larger for the High LAA participants (see Table 2 and Figure 4). Finally, we observed differences between the two groups of learners in short-range phase synchronisation in theta, alpha and gamma bands. The highly skilled learners exhibited larger increase of intracluster PS in time than the moderately skilled participants, each time in blocks 57 of the AGL task. The differences between the groups were localised in the right hemisphere, at the frontal electrodes for theta band, and centro-parietal electrodes for alpha and gamma bands (see Figure 5).

\section{Discussion and conclusions}

The goal of this study was to establish whether different levels of language aptitude influence the process of novel grammar learning and whether the possible differences in attainment of a new language can be traced back to brain oscillatory mechanisms recorded by means of EEG. To the best of our knowledge, only a handful of studies have investigated the temporal dynamics of EEG power and synchronisation properties during language acquisition so far. Neural oscillations have been shown to play a critical role in language processing, underpinning such aspects of it as speech perception (e.g. Giraud \& Poeppel, 2012; Pérez et al., 2015), syntactic operations (e.g. Bastiaansen et al., 2010) and semantic memory (e.g. Hanslmayr et al., 2016). Ubiquitous in the (human) brain, oscillatory mechanisms also potentially differ between individuals and such inter-individual differences may be coupled with various behavioural manifestations. In the case of the present experiment, we indeed established that cortical connectivity patterns and different 
profiles of spectral power modulations over time differentiated L2 learners with various levels of language analytical abilities.

Based on previous literature investigating the oscillatory mechanisms coupled with (language) learning, we hypothesised increases in the gamma band phase synchrony values as the learning proceeds and decreasing levels of synchronisation for the theta band. For the highly skilled learners, we expected stronger long-range gamma band synchrony than for average skilled learners. Lower analytical abilities were hypothesised to be reflected in high local gamma synchrony and power. Our results provided only partial confirmation of these hypotheses. First, increasing levels of global synchronisation values with learning were found for high frequency bands, both for gamma and beta bands alike. On the other hand, the synchronisation in slow frequency bands decreased as the learning proceeded. These results point to the importance of the synchronisation in alpha and theta bands in initial stages of learning. Since low-frequency phase synchronisation has been proposed to underlie long-range transmission of information (Clayton, Yeung, \& Cohen Kadosh, 2015; Weiss \& Mueller, 2012), our results suggest that such long-range transmission of information seems to be more prominent at the beginning of novel grammar learning. The observed increases in gamma and beta global PS values point to their importance for later stages of learning, and thus increasing reliance on localised neuronal activity as the learning proceeds.

Support for this claim comes from the results obtained from the long-range (intercluster) connectivity analysis. Although we did not observe any differences between our two groups of learners (with High and Average LAA), we did note that synchronisation between distant regions decreased over the course of the task in alpha and theta bands. The lack of significant differences between the two groups of learners in long-range phase coherence coupled with learning is in contrast with De Diego-Balaguer et al.'s (2011) finding that long-range gamma band coherence between frontal, temporal, and parietal regions accompanies successful language learning driven by rule extraction. Contrary to our predictions formulated on the basis of these previous findings, successful learners in our study could rather be distinguished by increased short-range phase synchronisation. De Diego-Balaguer et al. (2011) argued that the finding of increased long-range gamma band coherence is a marker of the process of binding neural representations to a global percept (i.e. not focussing attention on local features of the items). Since successful rule learning in our task similarly depended on attending to nonadjacent elements of the sequences, the lack of increased long-range gamma band phase synchrony among the highly skilled learners remains unclear.

By analysing global PS variations in four frequency bands using multilevel statistics, and within groups of electrodes chosen on the basis of their degree of bivariate PS, we showed that improving the task performance appears to be coupled with the development of whole-brain beta band coherence over time, and increases in local phase synchronisation values.

With regard to differences between the two groups of learners in global phase synchronisation values, the beta frequency band PS values proved to be predictive of the behavioural performance over time. This effect was modulated by the factor group: the behavioural scores of the highly skilled learners depended to a larger degree on their beta band synchronisation values over time than the behavioural scores of the average skilled learners. The oscillatory beta synchronization has been proposed to play an important role in language processing by - among others 
- underlying memory encoding and retrieval and supporting syntactically complex operations and high memory load demands (Weiss \& Mueller, 2012). Moreover, it has been hypothesised to "bind distributed sets of neurons into a coherent representation of (memorized) contents during language processing" (Weiss \& Mueller, 2012, p. 13) and to be suited to preserve neuronal activity (underlying the current sensorimotor or cognitive state) over time (Engel \& Fries, 2010). Our results add to this body of research on beta band frequency coherence and suggest that L2 grammar learning outcomes might - at least in part - be driven by timevarying whole-brain synchronisation within beta frequency range. What seems to be the case, is that communication between distributed neuronal assemblies in the beta frequency range supports the learning process and reflects the ability to acquire novel grammar.

Simultaneously to such whole-brain oscillatory activity, also local synchronisation mechanisms contribute to successful and efficient learning. Differences between High and Average LAA learners in terms of connectivity strength within certain groups of electrodes (intra-cluster connectivity) were observed in blocks 5-7 of the AGL task (see Figure 5). Evaluation of the behavioural learning curves of the two groups (see Figure 2) reveals that precisely at this time during the task, the High and Average LAA participants differed most in their performance: whereas both groups kept improving their performance in blocks 5-7, the scores obtained by the highly skilled learners increased notably more steeply. In terms of the differences between the groups on neural level in the time frame in question, they were localised in the right hemisphere, at the frontal electrodes for theta band, and centro-parietal electrodes cluster for alpha and gamma bands (see Figure 5).

Frontal theta band oscillations have been shown to be associated with plastic changes in learning and memory (cf. Voelker et al., 2016) and to be coupled with successful encoding of new information (cf. Begus, Southgate, \& Gliga, 2015), including motivationally significant stimuli (Gruber, Watrous, Ekstrom, Ranganath, \& Otten, 2013). Our data support these previous results and point to the importance of localised theta band oscillatory synchronisation for encoding of novel syntactic information. Furthermore, the localisation of this effect ties with results reported by De Diego-Balaguer \& Lopez-Barroso (2010) which concern language rule learning performance being reflected in the amplitude of an ERP P2 component recorded on the right frontal electrodes. New grammar learning also appears to be tied to increased synchronisation at the right centro-parietal site, both in the slow, alpha band and in fast, gamma band frequency. Increased alpha band synchronisation of the High LAA participants can be traced back to its role in 'top-down control', information transfer and - possibly - the storage and retrieval of memory representations from long-term memory (cf. Klimesch, Sauseng, \& Hanslmayr, 2007). As presented in section 1, gamma band has been shown to be involved in perceptual and attentional processing, episodic memory formation, and to play an important role in learning (cf. Herrmann et al., 2004; Miltner et al., 1999; Reiterer et al., 2009). Together, these results highlight the functional importance of the right regions for the grammar acquisition process and are in line with recent investigations into resting-state EEG power measurements prior to language learning by Prat, Yamasaki, Kluender and Stocco (2016). The authors found power in beta and low-gamma frequency ranges over right temporo-parietal regions to correlate positively with the rate of subsequent L2 learning and concluded that variability in functioning of the right hemisphere might relate to L2 learning rate. Such a conclusion is also consistent with results from our group concerning individual differences in grammar learning abilities investigated by 
means of fMRI (Kepinska et al., 2017). Employing a comparable design to the present one (adapted for MRI protocol), we found that during learning highly skilled learners could be distinguished from the average ones by increased activity localised in the right angular/supramarginal gyrus and right superior frontal and middle frontal gyri, and in the posterior cingulate gyrus. These recent experiments thus underscore the supporting role of the right hemisphere in language learning and suggest that engagement of the right hemisphere (localised both in the frontal and parietal sites) might lie at the centre of individual differences in L2 skills and attained proficiency (see Kepinska et al., 2016 for a further discussion on the importance of the right hemispheric involvement in grammar acquisition). In particular, in the present study, compared to the average skilled learners, the highly skilled learners exhibited increased synchronisation in theta band in right frontal areas, and a higher degree of synchronous oscillations within the right centro-parietal region. These mechanisms might possibly be underlying the differently realised learning curves by the two groups.

Although largely consistent with the localisation of neural activity coupled with L2 learning performance, the nature of the EEG signals recorded by our and Prat et al.'s (2016) study varies. Whereas Prat and colleagues reported values of spectral power in beta and low gamma frequency to correlate with L2 learning rate, our data provide information about localised synchronisation of the EEG signal marking increasing proficiency in a new language. As mentioned before, power within particular frequency bands measures the magnitude of oscillations over a specified time range, whereas coherence is an index of oscillatory coupling between two signals irrespective of their power (Kam, Bolbecker, O'Donnell, Hetrick, \& Brenner, 2013). Since Prat et al.'s data were recorded during resting state, prior to L2 learning, the power values most probably reflect the default neuronal state and its suitability for subsequent language learning. Our data, on the other hand, show that when the learning is actually taking place, there needs to be functional connectivity within particular neural assemblies in order for the learning to be successful.

With regard to the spectral power variations recorded during our experiment, we observed a significantly higher increase of values in the alpha band at the posterior electrodes among the highly skilled learners towards the end of the AGL task (in blocks 11-13), see Figure 3. Notably, in terms of power spectra, it was the only significant difference between the two groups of learners. According to one view present in the neurophysiological literature, the alpha rhythm results from an idle, relaxed state (cf. Berger, 1929; Pfurtscheller, Stancák, \& Neuper, 1996), and when recorded at posterior electrodes, it can reflect reduced attention or distraction (Clayton et al., 2015) ${ }^{4}$. Considering the fact that the performance on the AGL task of the highly skilled learners in blocks 11-13 was stable and still better than Average LAA group's performance (see Figure 2), the possible reduced attention seems not have had adverse consequences for the learning process. It might be the case that, at the period in question, the highly skilled participants have learned enough to perform well on the task, and were no more actively engaged in the

\footnotetext{
${ }^{4}$ As stated above (see the discussion on the interpretation of the increased alpha band coherence among the Highly skilled learners), reports also suggest that alpha activity plays an important role during active cognitive processes (cf. Klimesch et al., 2007). However, since the observed significant increase in the alpha power for the High as compared to Average LAA participants was constrained to posterior electrodes, our data rather seem to point to the classical 'cortical idling' interpretation.
} 
learning process as such. Analogous findings in the context of memory encoding were found by Park et al. (2014) who suggest that increase in alpha power during a memory task can reflect intentional inhibition and disengagement of taskirrelevant brain regions. Furthermore, such observations have also been made in the context of neural efficiency investigations by Jaušovec $(1996,2000)$. Neural efficiency theory - using fewer mental resources, in a more focused and goaldirected way, while dealing with demands of the task at hand (Neubauer \& Fink, 2009) - has been repeatedly employed in investigations examining the nature of neural processing in gifted individuals. In Jaušovec's studies (1996, 2000), the gifted individuals in comparison with average ones were reported to exhibit higher alpha power during problem solving, which was interpreted as less mental effort and disengagement of brain areas not required for the problem at hand. Our data suggest that participants exhibiting increased alpha power might have already learned all the necessary information and did not need to further focus their attention on the presented stimuli - a view following the idea that high degrees of language aptitude enable learners to attain high levels of proficiency in an L2 with relative ease and little time investment. The neural efficiency perspective might also shed light on the different profiles of global power evolvement over time in the high frequencies (beta and gamma). As presented in Figure 3, where the values of power among the highly skilled learners remained virtually unchanged, the average learners exhibited high increases in the values.

It is important to note that, as mentioned in Section 3.1, we used a common range (8-12 Hz) for defining the alpha band for all participants as it is customary in many EEG language studies (e.g., De Diego-Balaguer et al., 2011), instead of defining it based on individual alpha peaks. The drawback of our approach might be that a fixed frequency band may be unsuited to different individuals, due to age-related and/or participant-to-participant variability (see e.g., Hashemi et al., 2016), or for different conditions, as it has been showed that alpha peak frequency increases with cognitive demands (Haegens, Cousijn, Wallis, Harrison, \& Nobre, 2014). This could be solved by choosing participant or group-dependent frequency limits. However, this approach might have created another unwanted bias in our work, which targets group-level statistics, as different frequencies show different oscillatory characteristics. It might prove problematic to determine if the results are due to brain activity in the band of interest (e.g., alpha band) or due to differential background activity in different frequencies. In addition, both groups were age-matched, so age-related changes in alpha peaks are not an issue in our study. We did calculate the alpha peaks for all the individuals and made sure that in all cases they fell within the range we used. Thus, our approach can be regarded as an intermediate one: we selected the same frequency limits for all participants, rather than fixated these frequency limits to the true alpha range of our participant sample, which we believe is a reasonable trade-off between choosing the most realistic limits for the band of interest and ensuring comparability across groups. Nevertheless, this approach might be regarded as a limitation of our analysis and future studies should consider calculating the individual alpha frequency peaks and defining the relative boundaries for each individual and task phase in order to avoid any biases caused by a priori chosen boundaries.

A possible direction for future research concerns the way in which language aptitude, or the ease of acquiring new languages, can be modified. Such ideas have already been pursued in the context of semantic learning, where pharmacological substances such as levodopa have been shown to boost acquisition of novel lexicon and enhance consolidation of newly learned words (Knecht et al., 2004; Shellshear 
et al., 2015). Identification of brain regions related to language learning success, or increased efficiency in acquiring novel L2 materials, opens further possibilities for increasing learners' capabilities and offers foundations for probing the effects of non-invasive stimulation modulating neuronal activity patterns. As pointed out by Prat et al. (2016), power spectra of the EEG signal can indeed be modified. Simultaneously, there is increasing evidence for the possibility of non-invasive modulation of brain connectivity by means of transcranial current brain stimulation (see Luft, Pereda, Banissy, \& Bhattacharya, 2014 for a review). The present data, together with other research identifying brain regions underlying successful performance on language learning tasks (cf. Kepinska et al., 2016; Prat et al., 2016), provide a suggestion for a possible target of such stimulation in the form of the right parietal regions, and to some extend right frontal regions. Such stimulation might prove particularly effective in cases where the learning concerns novel grammatical rules.

In sum, by analysing EEG data recorded during a learning task, we have provided evidence for the functional role of neural oscillations in novel grammar acquisition. Having combined different measurements applied to the recorded signals, we offer a multi-layered picture of language learning and development of L2 knowledge on a neural level. In particular, on the basis of our results obtained with the global PS values and analyses investigating short- and long-range connectivity within the four frequency bands, we can conclude that oscillatory neural mechanisms underlying the grammar learning process and reflecting pre-tested analytical abilities operate on multiple levels, are topographically dispersed and dynamic in nature. Over the course of the task, the learning process seems to be driven by whole-brain functional connectivity between neuronal assemblies achieved by means of communication in the beta band frequency. On a shorter time-scale, increasing proficiency on a grammar learning task appears to be supported by stronger local synchronisation within the right hemisphere regions. Finally, we observed that the highly skilled learners might have exerted less mental effort, or reduced attention for the task at hand once the learning was achieved, as evidenced by the increasing alpha band power.

\section{Acknowledgements}

This research was supported by the Leiden University Centre for Linguistics and the NWO Graduate Programme; EP acknowledges the financial support from the Spanish MINECO through the project TEC2016-80063-C3-2-R. The funding sources had no involvement in the preparation of the manuscript, or in the decision to submit the article for publication. We thank Ferdi van de Kamp, Peter McCarthy, Fiona Patrick, Bobby Ruijgrok and Jos Pacilly for their help in data acquisition, and Bertram Opitz for advice on creation of the stimulus material, and to two anonymous reviewers for their insightful feedback on an earlier version of this manuscript.

\section{References}

Abrahamsson, N., \& Hyltenstam, K. (2008). The robustness of aptitude effects in near-native second language acquisition. Studies in Second Language Acquisition, 30(4), 481-509. http://doi.org/10.1017/S027226310808073X

Akaike, H. (1973). Information theory and an extension of the maximum likelihood principle. 2nd International Symposium on Information Theory, 267-281. 
http://doi.org/10.1016/j.econlet.2011.12.027

Allefeld, C., \& Bialonski, S. (2007). Detecting synchronization clusters in multivariate time series via coarse-graining of Markov chains. Physical Review E - Statistical, Nonlinear, and Soft Matter Physics, 76(6), 1-7. http://doi.org/10.1103/PhysRevE.76.066207

Allefeld, C., \& Kurths, J. (2004). An approach to multivariate phase synchronization analysis and its application to event-related potentials. International Journal of Bifurcation and Chaos, 417-426.

Bastiaansen, M., Magyari, L., \& Hagoort, P. (2010). Syntactic unification operations are reflected in oscillatory dynamics during on-line sentence comprehension. Journal of Cognitive Neuroscience, 22(7), 1333-1347. http://doi.org/10.1162/jocn.2009.21283

Begus, K., Southgate, V., \& Gliga, T. (2015). Neural mechanisms of infant learning: differences in frontal theta activity during object exploration modulate subsequent object recognition. Biology Letters, 11(5).

Benchenane, K., Peyrache, A., Khamassi, M., Tierney, P. L., Gioanni, Y., Battaglia, F. P., \& Wiener, S. I. (2010). Coherent theta oscillations and reorganization of spike timing in the hippocampal- prefrontal network upon learning. Neuron, 66(6), 921-936. http://doi.org/10.1016/j.neuron.2010.05.013

Berger, H. (1929). Über das Elektrenkephalogramm des Menschen. Archiv Für Psychiatrie Und Nervenkrankheiten, 87(1), 527-570. http://doi.org/10.1007/BF01797193

Bressler, S. L. (1995). Large-scale cortical networks and cognition. Brain Research Reviews, 20(3), 288-304.

Bressler, S. L., \& Menon, V. (2010). Large-scale brain networks in cognition: emerging methods and principles. Trends in Cognitive Sciences, 14(6), 277290.

Brod, G., \& Opitz, B. (2012). Does it really matter? Separating the effects of musical training on syntax acquisition. Frontiers in Psychology, 3(December), 543. http://doi.org/10.3389/fpsyg.2012.00543

Carroll, J. B. (1981). Twenty-five years of research in foreign language aptitude. In K. Diller (Ed.), Individual Differences and Universals in Language Learning Aptitude (pp. 83-118). Rowley, MA: Newbury House.

Clayton, M. S., Yeung, N., \& Cohen Kadosh, R. (2015). The roles of cortical oscillations in sustained attention. Trends in Cognitive Sciences, 19(4), 188195. http://doi.org/10.1016/j.tics.2015.02.004

Daly, I., Sweeney-Reed, C. M., \& Nasuto, S. J. (2013). Testing for significance of phase synchronisation dynamics in the EEG. Journal of Computational Neuroscience, 34(3), 411-432. http://doi.org/10.1007/s10827-012-0428-2

De Diego-Balaguer, R., Fuentemilla, L., \& Rodriguez-Fornells, A. (2011). Brain dynamics sustaining rapid rule extraction from speech. Journal of Cognitive Neuroscience, 23(10), 3105-3120. http://doi.org/10.1162/jocn.2011.21636

De Diego-Balaguer, R., \& Lopez-Barroso, D. (2010). Cognitive and Neural Mechanisms Sustaining Rule Learning From Speech. Language Learning, 60(s2), 151-187. http://doi.org/10.1111/j.1467-9922.2010.00605.x

de Graaff, R. (1997). The Experanto experiment: Effects of explicit instruction on second language acquisition. Studies in Second Language Acquisition, 19, 
$249-276$.

DeKeyser, R. M. (2000). The robustness of critical period effects In second language acquisition. Studies in Second Language Acquisition, 22, 499-533.

Dörnyei, Z., \& Skehan, P. (2003). Individual differences in second language learning. In C. J. Doughty \& M. H. Long (Eds.), The handbook of second language acquisition (pp. 549-630). Oxford: Blackwell Publishing.

Ellis, R. (2008). The Study of Second Language Acquisition (2nd Ed.). Oxford: Oxford University Press.

Engel, A. K., \& Fries, P. (2010). Beta-band oscillations-signalling the status quo? Current Opinion in Neurobiology, 20(2), 156-165. http://doi.org/10.1016/j.conb.2010.02.015

Finger, H., Bönstrup, M., Cheng, B., Messé, A., Hilgetag, C., Thomalla, G., ... König, P. (2016). Modeling of large-scale functional brain networks based on structural connectivity from DTI: comparison with EEG derived phase coupling networks and evaluation of alternative methods along the modeling path. PLOS Computational Biology, 12(8), e1005025. http://doi.org/10.1371/journal.pcbi.1005025

Frey, B. J., \& Dueck, D. (2007). Clustering by passing messages between data points. Science, 315(5814), 972-976.

Friederici, A. D., \& Singer, W. (2015). Grounding language processing on basic neurophysiological principles. Trends in Cognitive Sciences, 19(6), 1-10. http://doi.org/10.1016/j.tics.2015.03.012

Friederici, A. D., Steinhauer, K., \& Pfeifer, E. (2002). Brain signatures of artificial language processing: evidence challenging the critical period hypothesis. Proceedings of the National Academy of Sciences of the United States of America, 99(1), 529-534.

Giraud, A.-L., \& Poeppel, D. (2012). Cortical oscillations and speech processing: emerging computational principles and operations. Nature Neuroscience, 15(4), 511-517. http://doi.org/10.1038/nn.3063

Gollo, L. L., Mirasso, C., Sporns, O., \& Breakspear, M. (2014). Mechanisms of ZeroLag Synchronization in Cortical Motifs. PLOS Computational Biology, 10(4). http://doi.org/10.1371/journal.pcbi.1003548

Gruber, M. J., Watrous, A. J., Ekstrom, A. D., Ranganath, C., \& Otten, L. J. (2013). Expected reward modulates encoding-related theta activity before an event. NeuroImage, 64(6), 68-74. http://doi.org/10.1016/j.neuroimage.2012.07.064

Haegens, S., Cousijn, H., Wallis, G., Harrison, P. J., \& Nobre, A. C. (2014). Interand intra-individual variability in alpha peak frequency. NeuroImage, 92, 4655. http://doi.org/10.1016/j.neuroimage.2014.01.049

Hanslmayr, S., Staresina, B. P., \& Bowman, H. (2016). Oscillations and episodic memory: addressing the synchronization/desynchronization conundrum. Trends in Neurosciences, 39(1), 16-25. http://doi.org/10.1016/j.tins.2015.11.004

Harley, B., \& Hart, D. (2002). Age, aptitude, and second language learning on a bilingual exchange. In P. Robinson (Ed.), Individual differences and instructed language learning (pp. 302-330). Amsterdam: Benjamins.

Hashemi, A., Pino, L. J., Moffat, G., Mathewson, K. J., Aimone, C., Bennett, P. J., ... Sekuler, A. B. (2016). Characterizing Population EEG Dynamics 
throughout Adulthood. eNeuro, 3(6). http://doi.org/10.1523/ENEURO.027516.2016

Hauser, M. F. A., Hofmann, J., \& Opitz, B. (2012). Rule and similarity in grammar: Their interplay and individual differences in the brain. NeuroImage, 60(4), 2019-2026. http://doi.org/10.1016/j.neuroimage.2012.02.016

Herrmann, C. S., Munk, M. H. J., \& Engel, A. K. (2004). Cognitive functions of gamma-band activity: memory match and utilization. Trends in Cognitive Sciences, 8(8), 347-355. http://doi.org/10.1016/j.tics.2004.06.006

Hu, X., Ackermann, H., Martin, J. A., Erb, M., Winkler, S., \& Reiterer, S. (2013). Language aptitude for pronunciation in advanced second language (L2) learners: behavioural predictors and neural substrates. Brain and Language, 127(3), 366-376. http://doi.org/10.1016/j.bandl.2012.11.006

Ioannou, C. I., Pereda, E., Lindsen, J. P., \& Bhattacharya, J. (2015). Electrical brain responses to an auditory illusion and the impact of musical expertise. PLOS ONE, 10(6). http://doi.org/10.1371/journal.pone.0129486

Jaušovec, N. (1996). Differences in EEG alpha activity related to giftedness. Intelligence, 23(3), 159-173. http://doi.org/10.1016/S0160-2896(96)90001-X

Jaušovec, N. (2000). Differences in cognitive processes between gifted, intelligent, creative, and average individuals while solving complex problems: an EEG study. Intelligence, 28(3), 213-237. http://doi.org/10.1016/S01602896(00)00037-4

Kam, J. W. Y., Bolbecker, A. R., O’Donnell, B. F., Hetrick, W. P., \& Brenner, C. A. (2013). Resting state EEG power and coherence abnormalities in bipolar disorder and schizophrenia. Journal of Psychiatric Research, 47(12), 18931901. http://doi.org/10.1016/j.jpsychires.2013.09.009

Kepinska, O., de Rover, M., Caspers, J., \& Schiller, N. O. (2017). On neural correlates of individual differences in novel grammar learning: An fMRI study. Neuropsychologia, $\quad 98, \quad 156-168$. http://doi.org/10.1016/j.neuropsychologia.2016.06.014

Kiiski, H., Reilly, R. B., Lonergan, R., Kelly, S., O’Brien, M. C., Kinsella, K., ... Whelan, R. (2012). Only low frequency event-related EEG activity is compromised in multiple sclerosis: insights from an independent component clustering analysis. PLOS ONE, 7(9), e45536. http://doi.org/10.1371/journal.pone.0045536

Klimesch, W. (1999). EEG alpha and theta oscillations reflect cognitive and memory performance: a review and analysis. Brain Research Reviews, 29(2), 169-195. http://doi.org/10.1016/S0165-0173(98)00056-3

Klimesch, W., Sauseng, P., \& Hanslmayr, S. (2007). EEG alpha oscillations: The inhibition-timing hypothesis. Brain Research Reviews, 53(1), 63-88. http://doi.org/10.1016/j.brainresrev.2006.06.003

Knecht, S., Breitenstein, C., Bushuven, S., Wailke, S., Kamping, S., Flöel, A., ... Ringelstein, E. B. (2004). Levodopa: faster and better word learning in normal humans. Annals of Neurology, 56(1), 20-26. http://doi.org/10.1002/ana.20125

Kopell, N., Ermentrout, G. B., Whittington, M. A., \& Traub, R. D. (2000). Gamma rhythms and beta rhythms have different synchronization properties. Proceedings of the National Academy of Sciences of the United States of America, 97(4), 1867-1872. 
Luft, C. D. B., Pereda, E., Banissy, M. J., \& Bhattacharya, J. (2014). Best of both worlds: promise of combining brain stimulation and brain connectome. Frontiers in Systems Neuroscience, 8(132).

Macmillan, N. A., \& Creelman, C. D. (2005). Detection Theory: A User's Guide. Psychology Press.

Maguire, M. J., \& Abel, A. D. (2013). What changes in neural oscillations can reveal about developmental cognitive neuroscience: language development as a case in point. Developmental Cognitive Neuroscience, 6, 125-136. http://doi.org/10.1016/j.dcn.2013.08.002

Mardia, K. V., \& Jupp, P. E. (2000). Directional statistics. J. Wiley.

Meara, P. M. (2005). Llama language aptitude tests. Swansea, UK: Lognostics.

Mesulam, M. M. (1998). From sensation to cognition. Brain, 121(6), 1013-1052. http://doi.org/10.1093/brain/121.6.1013

Miltner, W. H., Braun, C., Arnold, M., Witte, H., \& Taub, E. (1999). Coherence of gamma-band EEG activity as a basis for associative learning. Nature, 397(6718), 434-436. http://doi.org/10.1038/17126

Mormann, F., Lehnertz, K., David, P., \& Elger, C. E. (2000). Mean phase coherence as a measure for phase synchronization and its application to the EEG of epilepsy patients. Physica D, 144(3), 358-369.

Neubauer, A. C., \& Fink, A. (2009). Intelligence and neural efficiency. Neuroscience and Biobehavioral Reviews, 33(7), 1004-1023. http://doi.org/10.1016/j.neubiorev.2009.04.001

Opitz, B., Ferdinand, N. K., \& Mecklinger, A. (2011). Timing matters: the impact of immediate and delayed feedback on artificial language learning. Frontiers in Human Neuroscience, $\quad 5$ (February), http://doi.org/10.3389/fnhum.2011.00008

Opitz, B., \& Friederici, A. D. (2003). Interactions of the hippocampal system and the prefrontal cortex in learning language-like rules. NeuroImage, 19(4), 1730-1737. http://doi.org/10.1016/S1053-8119(03)00170-8

Opitz, B., \& Friederici, A. D. (2004). Brain correlates of language learning: the neuronal dissociation of rule-based versus similarity-based learning. The Journal of Neuroscience: The Official Journal of the Society for Neuroscience, 24(39), 8436-8440. http://doi.org/10.1523/JNEUROSCI.2220-04.2004

Opitz, B., \& Friederici, A. D. (2007). Neural basis of processing sequential and hierarchical syntactic structures. Human Brain Mapping, 28(7), 585-592. http://doi.org/10.1002/hbm.20287

Park, H., Lee, D. S., Kang, E., Kang, H., Hahm, J., Kim, J. S., ... Jensen, O. (2014). Blocking of irrelevant memories by posterior alpha activity boosts memory encoding. Human Brain Mapping, 35(8), 3972-3987. http://doi.org/10.1002/hbm.22452

Pérez, A., Carreiras, M., Gillon Dowens, M., \& Duñabeitia, J. A. (2015). Differential oscillatory encoding of foreign speech. Brain and Language, 147, 51-57. http://doi.org/10.1016/j.bandl.2015.05.008

Pfurtscheller, G., Stancák, A., \& Neuper, C. (1996). Event-related synchronization (ERS) in the alpha band - an electrophysiological correlate of cortical idling: a review. International Journal of Psychophysiology, 24(1-2), 39-46. http://doi.org/10.1016/S0167-8760(96)00066-9 
Porz, S., Kiel, M., \& Lehnertz, K. (2014). Can spurious indications for phase synchronization due to superimposed signals be avoided? Chaos: An Interdisciplinary Journal of Nonlinear Science, 24(3), 33112. http://doi.org/10.1063/1.4890568

Prat, C. S., Yamasaki, B. L., Kluender, R. A., \& Stocco, A. (2016). Resting-state qEEG predicts rate of second language learning in adults. Brain and Language, 157-158, 44-50. http://doi.org/10.1016/j.bandl.2016.04.007

Psychology Software Tools. (2012). E-Prime. Pittsburgh, PA.

Qualtrics. (2013). Qualtrics. Provo, Utah.

Reiterer, S., Pereda, E., \& Bhattacharya, J. (2009). Measuring second language proficiency with EEG synchronization: how functional cortical networks and hemispheric involvement differ as a function of proficiency level in second language speakers. Second Language Research, 25(1), 77-106. http://doi.org/10.1177/0267658308098997

Reiterer, S., Pereda, E., \& Bhattacharya, J. (2011). On a possible relationship between linguistic expertise and EEG gamma band phase synchrony. Frontiers in Psychology, 2(November), 334. http://doi.org/10.3389/fpsyg.2011.00334

Robinson, P. (1997). Individual differences and the fundamental similarity of implicit and explicit adult second language learning. Language Learning, 47(1), 45-99.

Sawyer, M., \& Ranta, L. (2001). Aptitude, individual differences, and instructional design. In P. Robinson (Ed.), Cognition and Second Language Instruction (pp. 319-353). Cambridge University Press.

Schelter, B., Winterhalder, M., Timmer, J., \& Peifer, M. (2007). Testing for phase synchronization. Physics Letters, Section A: General, Atomic and Solid State Physics, 366(4-5), 382-390.

Shellshear, L., MacDonald, A. D., Mahoney, J., Finch, E., McMahon, K., Silburn, P., ... Copland, D. A. (2015). Levodopa enhances explicit new-word learning in healthy adults: a preliminary study. Human Psychopharmacology, 30(5), 341-349. http://doi.org/10.1002/hup.2480

Siegel, M., Donner, T. H., \& Engel, A. K. (2012). Spectral fingerprints of large-scale neuronal interactions. Nature Reviews Neuroscience, 13(2), 121. http://doi.org/10.1038/nrn3137

Singer, J. D., \& Willett, J. B. (2003). Applied Longitudinal Data Analysis. Applied Longitudinal Data Analysis: Modeling Change and Event Occurrence. Oxford University Press. http://doi.org/10.1093/acprof:oso/9780195152968.001.0001

Singer, W. (1993). Synchronization of cortical activity and its putative role in information processing and learning. Annual Review of Physiology, 55, 349374. http://doi.org/10.1146/annurev.physiol.55.1.349

Skehan, P. (2002). Theorizing and updating aptitude. In P. Robinson (Ed.), Individual Differences and Instructed Language Learning (pp. 69-93). Philadelphia/Amsterdam: John Benjamins Publishing Company.

Stam, C. J., Nolte, G., \& Daffertshofer, A. (2007). Phase lag index: Assessment of functional connectivity from multi channel EEG and MEG with diminished bias from common sources. Human Brain Mapping, 28(11), 1178-1193. http://doi.org/10.1002/hbm.20346 
Uhlhaas, P. J., \& Singer, W. (2010). Abnormal neural oscillations and synchrony in schizophrenia. Nature Reviews Neuroscience, 11(2), 100-113. http://doi.org/10.1038/nrn2774

Varela, F., Lachaux, J. P., Rodriguez, E., \& Martinerie, J. (2001). The brainweb: phase synchronization and large-scale integration. Nature Reviews Neuroscience, 2(4), 229-239. http://doi.org/10.1038/35067550

Voelker, P., Piscopo, D., Weible, A. P., Lynch, G., Rothbart, M. K., Posner, M. I., \& Niell, C. M. (2016). How changes in white matter might underlie improved reaction time due to practice. Cognitive Neuroscience, 1-7. http://doi.org/10.1080/17588928.2016.1173664

Wang, X.-J. (2010). Neurophysiological and computational principles of cortical rhythms in cognition. Physiological Reviews, 90(3), 1195-1268. http://doi.org/10.1152/physrev.00035.2008

Weiss, S., \& Mueller, H. M. (2003). The contribution of EEG coherence to the investigation of language. Brain and Language, 85(2), 325-343. http://doi.org/10.1016/S0093-934X(03)00067-1

Weiss, S., \& Mueller, H. M. (2012). "Too many betas do not spoil the broth": the role of beta brain oscillations in language processing. Frontiers in Psychology, 3, 201. http://doi.org/10.3389/fpsyg.2012.00201

Xiang, H., Dediu, D., Roberts, L., Norris, D. G., \& Hagoort, P. (2012). Language aptitude, working memory, and IQ in the perisylvian language network. Language Learning, 62(September), 110-130. 


\section{Supplementary materials}

\subsection{Details on the estimation of the PS indices}

Henceforth we provide a detailed explanation of the steps involved in the estimation of the PLV between two EEG electrodes.

\subsubsection{Estimation of the phases}

As a necessary initial step to estimate the PLV, we calculated the phases of each EEG channel at each frequency. Thus, we filtered each EEG channel, $x(t)$, in nonoverlapping narrow frequency bands of $2 \mathrm{~Hz}$ of bandwidth, and estimated the Hilbert Transform (HT) of each of these filtered signals, as follows:

$$
x(t)=\frac{1}{\pi} p \cdot v \cdot \int \frac{x(\tau)}{t-\tau} d \tau
$$

where p.v. stands for Cauchy's principal value. Then, the analytic, complex valued signal, is defined as

$$
x_{a}(t)=x(t)+i x(t)
$$

The phase of this complex-valued signal, $\phi_{x}(t)$, is then simply defined as:

$$
\phi_{x}(t)=\operatorname{arctag} \frac{\hat{x}(t)}{x(t)}
$$

\subsubsection{Estimation of bivariate PS}

For every pair of electrodes $x_{l}(t)$ and $x_{k}(t)(l, k=1, . ., 32 ; l \neq k)$ and each narrow frequency band, one can estimate the degree of PS between the corresponding analytic signals by the following index, termed phase locking value (PLV), or mean phase coherence (Mormann et al., 2000):

$$
P L V_{l k}(f)=\frac{1}{N}\left|\sum e^{-i \varphi_{l, k, f}(t)}\right|
$$

where $N$ is the total number of phases, $f$ is the centre frequency of each band and I I stands for the module of this complex number. Defined in this way, PLV ranges between 0 (no coherence) and 1 (perfect phase coherence). Yet, it is well-known (see, e.g., Daly, Sweeney-Reed, \& Nasuto, 2013) that this index can be different from zero only due to properties of the individual signals such as its short length. Thus, we used the formula suggested by (Mardia \& Jupp, 2000) to calculate the $p$ value for the null hypothesis that (4) was actually not different from 0 and set to 0 if $p<0.0001$ (i.e., at the $p>0.05$ level after applying Bonferroni correction for multiple comparisons for every pair of electrode and frequency band). The MATLAB scripts for the estimation of the phases, the PLV and its significant level are publicly available, as described in (Niso et al., 2013; García-Prieto, Bajo \& Pereda, 2017). 
Of note, using more sophisticated strategies to estimate the significance of the PLV entails the construction of surrogate data from the original EEGs, namely time series that share with the original EEGs most of their individual features but are independent between them by construction (Daly et al., 2013; Schelter, Winterhalder, Timmer, \& Peifer, 2007). The original PLV values are later compared to the distribution of "surrogated" PLVs, and the significance of the former is decided based on how different it is from the latter, using either parametric or non-parametric hypothesis test. Besides its computational complexity, there is no such thing as a perfect surrogate data in the literature, and the hypothesis tests provide $p$ values that are no lower than $1 / N_{s}$, where $N_{s}$ is the number of surrogates. Thus we decided to use the formula above, which has also the advantage to provide an analytic estimation of the $p$ value directly from the PLV.

Finally, PLV for each of the same four frequency bands as defined in the power spectral analysis was equally obtained by averaging across all the narrow frequency bands between the corresponding limits.

It may be argued that, for the case of scalp EEG data, PLV is known to be sensitive to volume conduction effects (Stam et al., 2007), so that other indices such as the phase lag index (PLI) or the imaginary part of the coherence function (Nolte et al., 2004) would be more appropriate in the present framework, as they disregard phase relationship with zero lag, as those due to volume conduction. Yet, on the one hand, recent results have shown that results from PLV are sometimes easier to interpret in neurophysiological terms than those from the PLI (Ioannou, Pereda, Lindsen, \& Bhattacharya, 2015; Porz, Kiel, \& Lehnertz, 2014). And, on the other hand, it is also known that a considerable amount of functionally relevant synchrony takes place with near zero or zero-phase lag (Finger et al., 2016; Gollo, Mirasso, Sporns, \& Breakspear, 2014). Further, this index has been used successfully to study the oscillatory encoding of second language (Pérez et al., 2015; Reiterer et al., 2011).

\subsection{Global PS values over time}

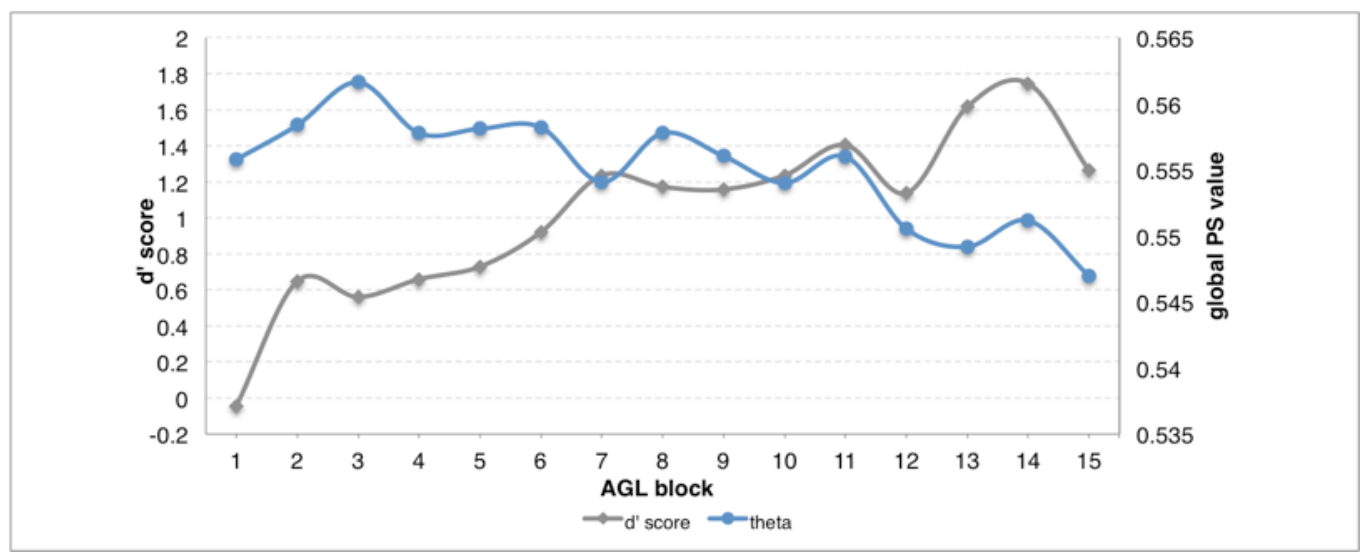



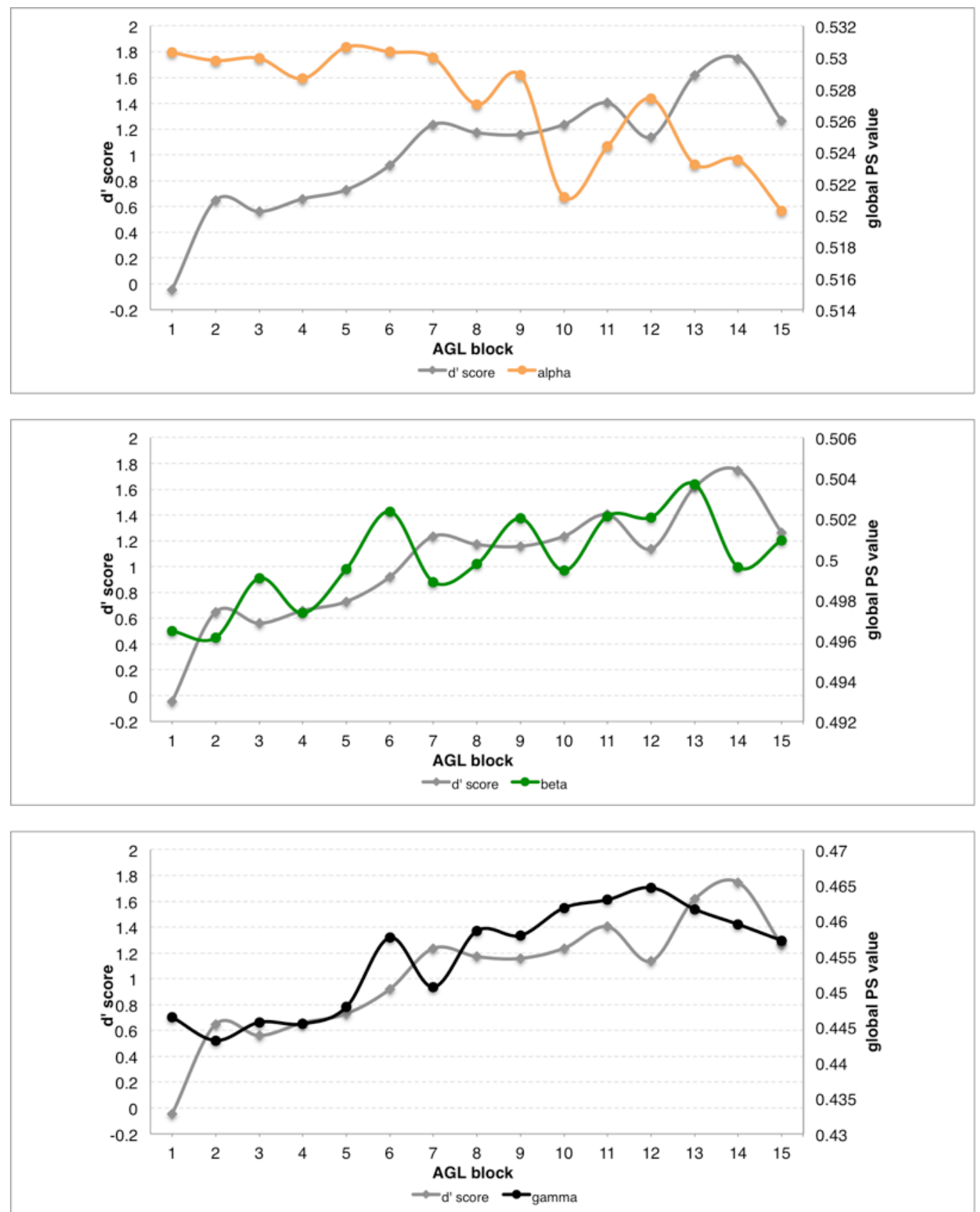

Supplementary Figure 1. Global PS (PLV) values in the four frequency bands (theta, alpha, beta, gamma) recorded during the fifteen AGL task blocks plotted against the respective mean AGL task scores. 